\title{
Psychological Problems of the Nursing Staff in COVID-19 Pandemic: A Systematic Review
}

\author{
Batoul Khoundabi ${ }^{1}$, Ahmad Soltani ${ }^{2}$, and Milad Ahmadi Marzaleh ${ }^{3,}$ \\ ${ }^{1}$ Assistant Professor of Biostatistics, Iran Helal Institute of Applied-Science and Technology, Research Center for Health Management in Mass Gatherings, Red \\ Crescent Society of the Islamic Republic of Iran, Tehran, Iran \\ ${ }^{2}$ Assistant Professor, Iran Helal Institute of Applied-Science and Technology, Research Center for Emergency and Disaster Resilience, Red Crescent Society of the \\ Islamic Republic of Iran, Tehran, Iran \\ 3 Department of Health in Disasters and Emergencies, School of Management and Medical Informatics, Shiraz University of Medical Sciences, Shiraz, Iran
}

* Corresponding author: Milad Ahmadi Marzaleh, Department of Health in Disasters and Emergencies, School of Management and Medical Informatics, Shiraz University of Medical Sciences, Shiraz, Iran. Tel: +987132340774; Fax: +987132340039; Email: miladahmadi marzaleh@yahoo.com

\section{Received 2021 March 13; Revised 2021 April 27; Accepted 2021 May 21.}

\begin{abstract}
Background: Given the fact that nurses are at the frontier of taking care of COVID-19 patients, they are directly or indirectly faced with many psychological problems.

Objectives: The present research used a systematic review approach to explore the psychological problems of nurses during the COVID19 pandemic in 2020 .

Methods: This systematic review was conducted using PubMed, Cochran Library, Scopus, EMBASE, Science Direct, ProQuest, and Web of Science databases until May 21, 2020. These databases were searched using some keywords and the papers with a focus on the psychological problems of nurses during the COVID-19 pandemic were selected.

Results: Primarily, the academic papers were searched using their titles, abstracts, and full texts. In total, 10 articles were selected for the final analysis; nine of them were cross-sectional in type and one of them was qualitative and phenomenological. Moreover, seven studies were carried out in the Chinese context and were followed by other similar studies performed in Italy, Pakistan, India, and Singapore. Based on the findings, the most prevalent psychological problems were panic, anxiety, stress, sleep disorders, discomfort, depression, lack of self-control, overstimulation, and post-traumatic stress disorder.

Conclusion: During the epidemic and pandemic of infectious diseases, such as COVID-19, a wide range of psychological problems arise among the healthcare staff, especially nurses. As nurses are at the frontier of taking care of and treating COVID-19 patients, they need to be fully supported. Healthcare policymakers should devise educational and psychological supporting protocols to improve the mental health of nurses.
\end{abstract}

Keywords: COVID-19, Health, Mental health, Nurses, Psychological

\section{Background}

Severe acute respiratory syndrome Coronavirus 2 (SARS-CoV-2) has prevailed in many countries worldwide since the World Health Organization (WHO) declared a COVID-19 pandemic on March 11, 2020 (1). The pandemic has assayed the resilience of healthcare systems, including hospitals, which were mostly not prepared for a worldwide pandemic. Readiness in dealing with this crisis has been required not only from medical facilities and equipment but also to healthcare staff treating patients with COVID-19 physically and mentally (2).

The health staff, including physicians, nurses, and other healthcare workers, have been faced with this critical situation. Healthcare workers at the frontline have been directly involved in the diagnosis, treatment, and care of patients with COVID-19. Hence, they have been at a higher risk of developing psychological distress and other mental symptoms (3). Healthcare workers have also experienced different types and symptoms of depression, anxiety, insomnia, and distress (4). Besides, daily reports on the COVID-19 epidemic, specifically regarding the number of deceased frontline medical staff, might add to the concerns of families (5).

The importance of mental health for the medical staff and its impact on the improvement of patient care has been investigated before in the case of epidemic diseases, such as SARS (6-8). The factors associated with the mental health of healthcare staff influenced by the COVID-19 epidemic and the resultant psychological states have also been explored in a few published studies $(8,9)$. Results of a study demonstrated that $42.0 \%$ of the physicians working in the mainland of China experienced very high levels of accumulated fatigue (10). Conduction of a comprehensive study on the mental health and challenges of healthcare workers during the COVID19 pandemic and exploration of the effective factors in mental health and psychological needs of healthcare professionals can help better manage the needs of this substantial population.

\section{Objectives}

The present systematic review was conducted on relevant papers in a number of major databases to summarize and discuss the aforementioned mental challenges during the pandemic. 


\section{Methods}

\subsection{Inclusion criteria and search strategies \\ 3.1.1. Search strategy}

The first step was registering the research protocol in the International Prospective Register of Systematic Reviews database (CRD42020187653). The research was a systematic review and the Preferred Reporting Item for Systematic Reviews and Meta-analyses (PRISMA) guideline was followed. The systematic review was performed on the papers published before May 21, 2020. The peer-reviewed papers that were written in English and aimed to answer "What psychological problems are nurses faced with during COVID-19?" were selected.

A quick and general search was initially carried out in Cochrane Library to ensure that there was no previous systematic review on this topic. When the gap appeared, the search was extended to electronic databases, such as PubMed, Scopus, EMBASE, Science Direct, Web of Science, Cochrane Library, and ProQuest. It is noteworthy that grey literature was searched as well, including books, websites, conference papers, and theses. The operator AND was used to search for keywords or phrases that were considered distinct. To search for synonymous keywords, the operator OR was employed. For the purposes of the study, all the keywords, abstracts, and titles of academic papers were searched. Mesh terms were used in the PubMed database to search for the relevant works. The search strategies are summarized in Table 1.

As there was no control group in the present research, no $\mathrm{C}$ or comparison group was specified in the patient/population, intervention, comparison, and outcomes (PICO) process. The keywords were selected by the researcher and finally, the relevant factors were extracted from the selected papers. In the next step, a comprehensive list of references of all papers was made, and their titles were examined by the researcher. Afterward, the articles that were not relevant to the research objective were excluded. To be on the safe side, all search procedures were repeated. It should be mentioned that EndNote-X9 was used to manage the sources.

\subsubsection{Inclusion criteria}

The papers were screened based on the expression of the psychological problems of nurses during COVID-19 in titles, keywords, and abstracts. To this end, first, the titles were scrutinized; afterward, the abstracts and full texts were reviewed. The systematic review was conducted on the papers published until May 21, 2020. Unpublished materials (grey literature) from reliable sources, such as protocols, conference papers, instructions, guidelines, and reports of credible organizations, were reviewed as well. The review articles as well as qualitative and quantitative papers were also included. The keywords were searched in the titles, abstracts, and keywords of papers. Additionally, the papers had to be relevant to the research question at hand.

\subsubsection{Exclusion criteria}

The papers that were not relevant to the research questions were excluded from the study.

\subsubsection{Screening}

At first, the titles of all papers found in the databases were examined and those that were relevant to the research question were selected. Secondly, the abstracts were reviewed by the researcher and those that were fully consistent with the purpose of the research and met the inclusion criteria were selected and their full-texts were read. Finally, the papers that focused on the psychological problems of nurses during the COVID-19 pandemic were included in the study. The papers were evaluated based on the PRISMA guideline. Citation and publication bias were also taken into account, and papers with a high citation count were examined closely. All the above-mentioned procedures were followed twice.

\subsubsection{Data extraction}

The papers were carefully reviewed and the required information was abstracted and extracted according to a specific form, which included the title, corresponding author, research sample, time and place of the research, research design, methodology, results, and conclusion. The abstraction forms were completed for all papers. All forms were analyzed by two researchers independently and the results were tabulated. Moreover, in case of any contradictions, other researchers were consulted. The final forms were completed in Microsoft Word Office (version 2016).

\begin{tabular}{lcc}
\hline \multicolumn{2}{c}{ Table 1. Search strategies used to explore the psychological problems of nurses during COVID-19 } \\
\hline PIO & \#1 AND \#2 AND \#3 & Strategy \\
\hline P & Nurse OR Nursing Personnel OR Registered Nurses OR Health Personnel OR Health worker OR Health caregiver & \#1 \\
I & Severe acute respiratory syndrome coronavirus 2 OR Coronavirus OR SARS-CoV-2 OR COVID-19 OR COVID19 & OR New coronavirus OR Corona \\
O & Mental OR Psychological OR Mental Disorders OR Mental health OR Psychic OR Psychologic OR Stress OR & Anxiety OR Depression
\end{tabular}

PIO: patient/population, intervention, and outcomes 


\section{Results}

The initial search led to the extraction of 809 papers, 235 of which were excluded as they recurred in the databases. Afterward, 574 titles were analyzed and 452 relevant papers were excluded as they did not match the purpose of the research. The abstracts of the 122 papers found in the previous step were analyzed and 101 papers were excluded as they were not related to the purpose of the research. Finally, 16 full texts were selected, 10 of which were totally in line with the research objectives. The final selection diagram of the papers is depicted in Figure 1.

Table 2 summarizes the types of the extracted articles. Accordingly, nine studies were crosssectional and one was qualitative using a phenomenological approach.

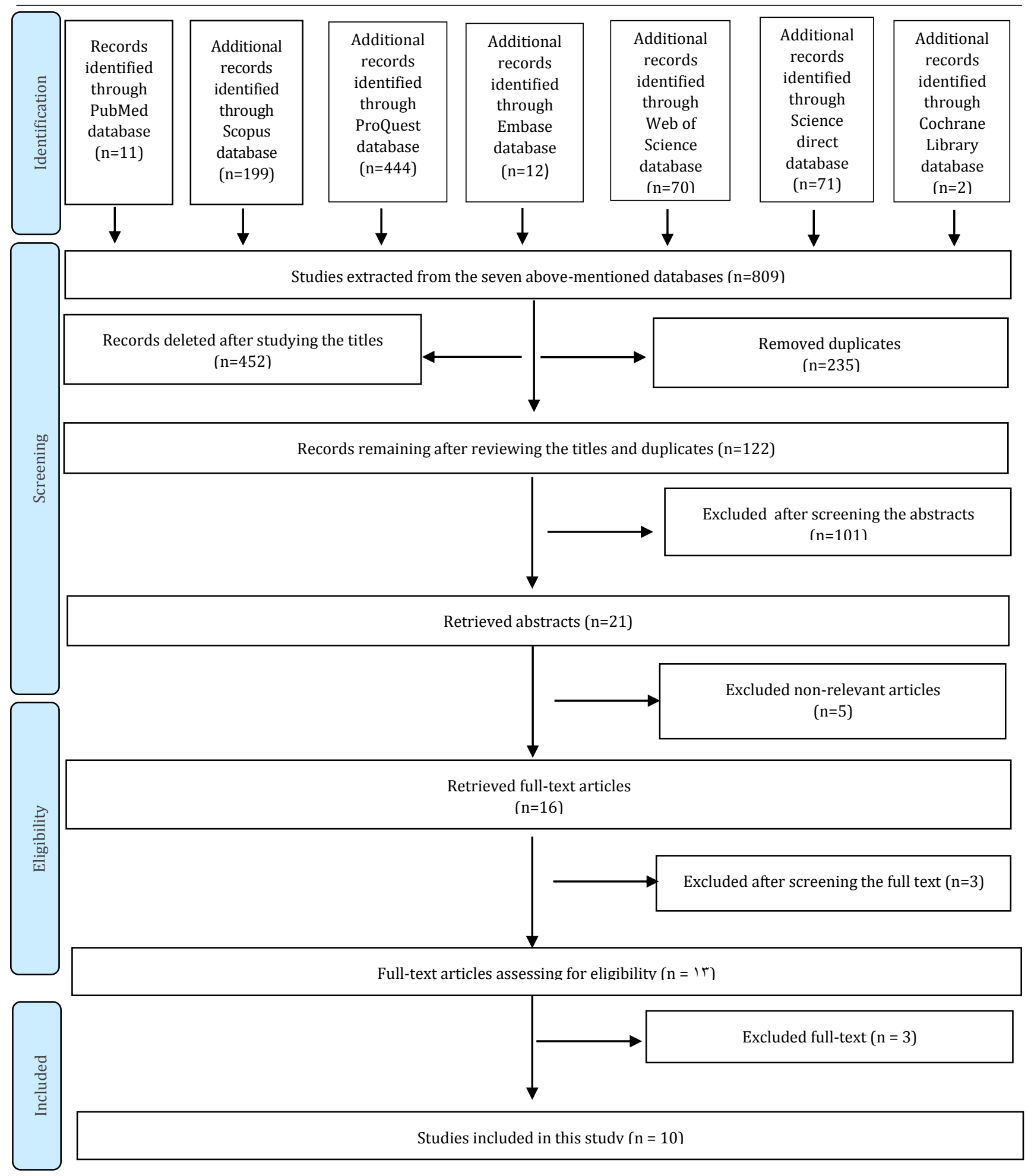

Figure 1. Preferred reporting item for systematic reviews and meta-analyses flow diagram of screened papers 
Khoundabi B et al.

\begin{tabular}{lccc}
\hline \multicolumn{2}{l}{ Table 2. Types of the selected academic papers } & & \\
\hline Research type & Percentage & Number & Reference (s) \\
\hline Qualitative & 10 & 1 & $(7)$ \\
Cross-sectional & 90 & 9 & $(3,6,8,9,11-15)$ \\
\hline
\end{tabular}

The contents of the selected 10 papers have been summarized in Table 3. The information included the title, corresponding author, place (country) and time of the research, purpose of the research, sample, design, methodology, results, and conclusion.

\begin{tabular}{|c|c|c|c|c|c|c|c|}
\hline \# & $\begin{array}{c}\text { Corresponding } \\
\text { author/title }\end{array}$ & Country & Year & Purpose & Sample & Design & Methods \\
\hline 1 & $\begin{array}{c}\text { Niuniu Sun et al./A } \\
\text { Qualitative Study } \\
\text { on the } \\
\text { Psychological } \\
\text { Experience of } \\
\text { Caregivers of } \\
\text { COVID-19 Patients }\end{array}$ & China & $\begin{array}{c}\text { January } \\
20- \\
\text { Februar } \\
\text { y } 10 \\
(2020)\end{array}$ & $\begin{array}{l}\text { Discovering } \\
\text { psychological } \\
\text { experiences of } \\
\text { nurses while } \\
\text { taking care of } \\
\text { COVID-19 } \\
\text { patients }\end{array}$ & $\begin{array}{l}20 \text { nurses } \\
\text { working actively } \\
\text { in the first } \\
\text { university } \\
\text { hospital of } \\
\text { Henan }\end{array}$ & $\begin{array}{c}\text { Qualitative- } \\
\text { phenomenology }\end{array}$ & $\begin{array}{l}\text { Face-to-face or on- } \\
\text { phone semi- } \\
\text { structured } \\
\text { interviews with } \\
\text { nurses; data analysis } \\
\text { via Colaizzi's method }\end{array}$ \\
\hline
\end{tabular}
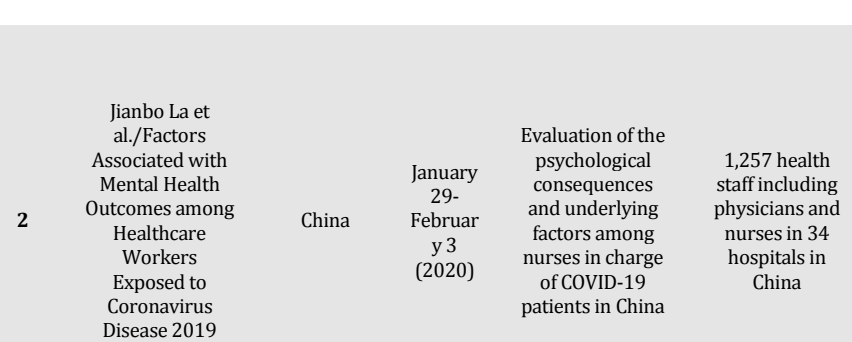

Nicholas WS
Chew/A

Multinational,

Multicenter Study on the

Psychological

Outcomes and

Associated

Physical Symptoms

amongst

Workers during

COVID-19

Outbreak

\begin{tabular}{|c|c|c|c|}
\hline $\begin{array}{l}\text { Februar } \\
\text { y } 19- \\
\text { April } 17 \\
(2020)\end{array}$ & $\begin{array}{l}\text { To explore the } \\
\text { correlation } \\
\text { between } \\
\text { psychological } \\
\text { outcomes and } \\
\text { physical } \\
\text { symptoms } \\
\text { among } \\
\text { healthcare staff }\end{array}$ & $\begin{array}{l}906 \text { participants } \\
\text { from the health } \\
\text { staff, including } \\
\text { physicians, } \\
\text { nurses, and } \\
\text { managers in five } \\
\text { hospitals of } \\
\text { India and } \\
\text { Singapore; } \\
\text { number of } \\
\text { nurses=355 }\end{array}$ & Cross-sectional \\
\hline
\end{tabular}

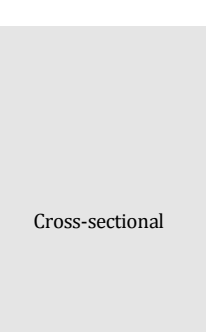

Symptoms of depression, anxiety, insomnia, and distress were tested through the patients ealth questionnair (nine items), generalized anxiety disorder scale (seve items), severity of insomnia (seven items), and impact of event scale $(22$ items); use of multivariate regression analysis

Use of a self-rating questionnaire containing several parts, such as demographic

information, medica background, prevalence of symptoms in the recent months, Depression Anxiety Stress Scales-21, and IES-R; analysis of the correlation between physical symptoms and psychological and psychological consequences, such
as depression, as depression,
anxiety, stress, and nxiety, stress,
PTSD

Use of the PGWBI (30 multiple-choice tems) to explore the psychological healt of the medical staff (e.g. nurses and physicians) treating Use of a researchermade questionnair (20 items) scored based on a four-point Likert scale, use of Self-Rating Anxiety Scale to testanxiety, Scale to test anxiety,
use of Self-Rating use of Self-Rating test depression use test depression, use of WeChat to send the questionn
online
Conclusion

Categorization of the psychological experiences of nurses into four themes: 1. emergence of negative feelings in the initial stages, including fatigue, discomfort, panic, and anxiety about oneself and one's family, 2. self-exposure strategies, such as psychological strategies, such as psychological
adaptation, team support, and logical adaptation, team support, and logical cognition, 3. under-pressure growth,
such as increased professional such as increased professional
responsibility and self-reflection, and 4 . responsibility and self-reflection, and 4
positive effects (all participants positive effects (all participants expressed anxiety about the patients in isolation, 15 nurses experienced anxiety, $50 \%$ of the nurses experienced tension, all nurses felt anxious about themselves and their families

765 participants (60.8\%); $50.4 \%$ diagnosed with depression, $44.6 \%$ with anxiety, $34 \%$ with insomnia, and $71.5 \%$ with distress. Female nurses at the frontier fighting the disease showed the most symptoms in Wuhan China the same group showed the highest risks same group showed the highest risks of mental disorders, such as depression (OR=1.52, 95\% Cl $=1.11-2.09, \mathrm{P}=0.01$ ) anxiety $(\mathrm{OR}=1.57,95 \% \mathrm{Cl}=1.22-2.02$, $\mathrm{P}<0.001$ ), insomnia (OR=2.97, 95\% $\mathrm{Cl}=1.92-4.60, \mathrm{P}<0.001)$, and distress
$(\mathrm{OR}=1.60,95 \% \mathrm{Cl}=1.25-2.04, \mathrm{P}<0.001)$

Mean IES-R score: 8.29 \pm 9.79 ; anxiety and overstimulation found as common symptoms; 48 participants $(5.3 \%)$ diagnosed with moderate to severe depression; 79 participants $(8.7 \%)$ diagnosed with moderate to sever anxiety; 20 participants $(2.2 \%)$ diagnosed with moderate to severe stress; 34 participants (3.8\%) diagnosed with moderate to severe symptoms of distress; $33 \%$ diagnosed with headache; occasional cases of PTSD symptoms among nurses;

homogenizing participants in terms of age and ender showed: depression $(\mathrm{OR}=2.79,95 \% \mathrm{CI}=1.54-5.07, \mathrm{P}=0.001)$ (OR $=2.79,95 \% \mathrm{Cl}=1.54-5.07, \mathrm{P}=0.001)$
anxiety $(\mathrm{OR}=2.18,95 \% \mathrm{CI}=1.36-3.48$, $\mathrm{P}=0.001)$, stress $(\mathrm{OR}=3.06,95 \%$
$\mathrm{C}=1.36-3.48$ $\mathrm{P}=0.001)$, stress $(\mathrm{OR}=3.06,95 \%$
$\mathrm{CI}=1.27-7.41, \mathrm{P}=0.13)$, and PTSD (OR=2.20, 95\% CI=1.12-4.35, $\mathrm{P}=0.023)$; emergence of physical symptoms along with psychological symptoms

Fear of the disease as the main cause of mental and psychological symptoms; mean score of psychological effects=16.4, depression=21.4, negative discomfort=28.1, lack of selfcontrol $=26.23$, vitality $=26.44 \cdot \mathrm{mea}$ scores of PGWBI were 0.924,0.614, and 0.5 for the age groups of $20-30,31-40$ 0.5 for the age groups of $20-30,31-40$,
and above 50 years old

At the peak of the epidemic, positive and negative feelings developed in nurses. At the initial stages, the latter emerged sooner than the former. Self-

exposure styles and psychological growth played a key growth played a key role in the mental
health of nurses.

The medical staff o the hospitals in Wuhan, China, especially female nurses who were directly involved in the diagnosis, care the diagnosis, car provision, and treatment of COVI

19 patients, wer under more psychological
pressure. pressure

A statistically significant correlation was

found between the prevalence of physical and psychological symptoms among
the healthcare staff. the healthcare staff
Psychological interventions were
found to be effective in treatment

The prevalence rates of anxiety and depression symptoms among nurses were $27.9 \%$ and $43 \%$, respectively. Depression and anxiety background were found as the risk factors: anxiety ( $\mathrm{T}=-3.635, \mathrm{P}=0.000,95 \% \mathrm{CI}=-16.360 \sim$

4.7), depression ( $\mathrm{T}=-2.835, \mathrm{P}=0.005$, $95 \% \mathrm{Cl}=-18.238 \sim-3.254)$.
The government is expected to provid the required

facilities for nurses and medics to guarantee their psychological health

Frontier medical staff fighting COVID19 showed symptoms of high depression and anxiety. Promotio of adaptive capacities to psychological symptoms can reduce the negative feelings. 
Rodolfo Rossi et al./Mental Health Outcomes among Front and Second Line Health Workers

Associated

with the COVID-19

Pandemic in Italy

\begin{tabular}{|c|c|c|c|}
\hline \multirow{4}{*}{ Italy } & & $\begin{array}{l}\text { To report the } \\
\text { psychological }\end{array}$ & $\begin{array}{c}472 \text { nurses, } 433 \\
\text { specialists, } 86 \\
\text { general }\end{array}$ \\
\hline & March & of the COVID-19 & practitioners, \\
\hline & 31 & epidemic among & 112 nurse \\
\hline & (2020) & $\begin{array}{c}\text { Italian } \\
\text { healthcare staff }\end{array}$ & $\begin{array}{l}\text { assistants, and } \\
275 \text { others }\end{array}$ \\
\hline
\end{tabular}

Cross-sectional
To explore the psychological effects of COVID 19 on the frontier medical staff fighting the disease and the coping strategies in Hunan, the adjacent
proving adjacent
province to province to
Hubei, during Hubei, during
the epidemic of the disease
534 health staff

(248 nurses, 233 specialists, 48 specialists, 48
medical

technicians, and 5 hospital workers

Coronavirus

Disease 2019

(COVID-19) in

Hubei, China
Online submission of

questionnaires in social networks; now-ball sampling mental pressure, 1
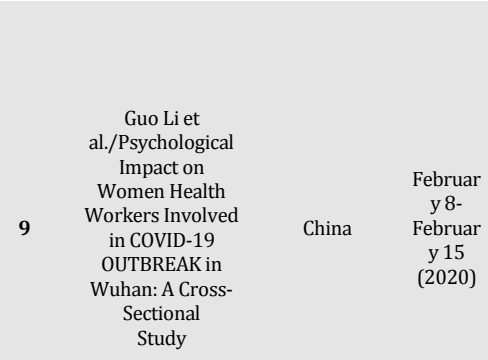

To explore the immediate psychological healthcare
hects on fema workers in Tongii; determine the predictors of acute stress, depression, and anxiety; explore the sources of acute stres content of the questionnaires formation, feature of workplace, information about direct exposure to COVID-19, and

exposure to infected colleagues; main psychological effects: symptoms of stress disorder, PTSD, severe depression anxiety, insomnia, and perceived stres

Use of a questionnaire containing five part and 67 items

(score=0-3): 14

items exploring how

the staff felt during the COVID-19 pandemic, 19 item exploring the potential factors accounting for items exploring stress management 11 items identifying personal or stresscoping strategies, 9 items exploring whatever that can make the staff feel make the staff feel in future

Snowball sampling online survey via WeChat, email, or phone calls with subjects in differen provinces of China; inclusion criteria: 1 . healthcare staff, 2 . 18-60 years of age, 3 .

full comprehensio of the research of the research questions, 4 . no
symptoms of mental disorders, such as disorders, such as
depression, anxiety and PTSD
The observed symptoms: PTSD, severe depression, anxiety, insomnia, and stress in $49.38 \%, 24.73 \%, 19.80 \%$ $8.27 \%$ and $21.9 \%$ of the staff, respectively. Regression test results: lower age and female gender had correlations with all adverse effects, except for insomnia; higher odds of PTSD in general practitioners; higher exposure of nurses and nurse assistants to severe insomnia; strong correlation between having a deceased hospitalized, or quarantined friend or relative and PTSD, depression insomnia, and stress symptoms; correlation between exposure to correlation between exposure to
infection and emergence of depression symptoms

First part: higher levels of anxiety of higher tendency of technicians to be well-recognized $(\mathrm{P}<0.001)$; second part: anxiety about personal health as the main factorinvolved in stress (P<0.001) which showed to be more $(P<0.001)$ The $30-41$ tors bevalent in whe part: family health as the most effective factor in reducing the stress of the staf $(\mathrm{P}<0.05)$ with no difference among various age groups; fourth part:

appealing for help from family and friends as a key supportive act

$(\mathrm{P}<0.001)$; medical staff's expression of no particular effect of consultation with a psychologist on the reduction of stress; fifth part: availability of detaile instructions on infection control, specialized equipment, and fewer case of COVID-19 affliction as a relief

Prevalence of PTSS one month after COVID-19 pandemic in $3.8 \%$ of healthcare workers, more vulnerability

of female healthcare workers $(\mathrm{OR}=$

2136, CI=1388), more stimulation symptoms among the healthcare workers who are more exposed ( $\mathrm{OR}=4.026, \mathrm{Cl}=1.233,13.140)$, statistically significant difference between the participants with an without PTSS $(\mathrm{P}=0.001)$ and also between groups with different exposure frequencies $(\mathrm{P}=0.026)$

Low weight induced by depression, anxiety, and acute stress observed in $14.2 \%, 25.2 \%$, and $31.6 \%$ of subjects, respectively (among female healthcare workers); drinking background found as a key factor in anxiety $(\mathrm{OR}=1.95)$ and depression symptoms ( $\mathrm{OR}=1.71)$;

having a positive or suspicious case in family members found as a factor involved in anxiety ( $\mathrm{OR}=1.31$ ); referring to the nursing or medical technician ward and having a positive or suspected case among family members found to be a risk factor of acute stress ( $O R=1.41$ and 1.39 , respectively); working in and 1.39 , respectively); working in
other wards found as a protective factor for acute stress (OR=0.78) nurses about financial issues $(\mathrm{P}=0.002)$

A vast majority of healthcare staff fighting COVID-19, especially young women, suffer fro psychological problems and are mentally unhealthy. It is essential to have better monitoring of certain interventions

for health staff ror to pandemic.

Prevalence of COVID-19 in Hubei increased stress among the medical staff in adjacent provinces (i.e. Hunan). It seems essential that the efforts of the healthcare staff be continuously acknowledged by the hospital management and the government, guidelines on how to control infection be

developed,

specialized

equipment be

provided for

infection

management, and

medical staff be appreciated for their prospective efforts.

Reaction to perceived mental health is a medical emergency that can also help healthcare workers and society to prepare themselves to react appropriately to events.

Consulting with psychologists helps policymaking and relevant supportive acts to solve the psychological problems of women It also helps manage the crisis more efficiently and face health consequences in the long run. above 8 for GAD-7 of onn-Whitney U test for

Use of an onlin survey: Chinese version of Signs of Stress and the SelfRating Anxiety Scale. Use of descriptive correlational methods, univariate correlation analysis, and multivariate regression analysis
The mean scores of Signs of Stress and Self-Rating Anxiety Scale were found to be $39.9 \pm 12.92$ and $32.19 \pm 5.5$ respectively, and a significant positive correlation was observed between the two $(r=0.676, \mathrm{P}<.05)$. According to multivariate regression analysis, being an only child, work hours per week, and anxiety were the main predictors of nurses' stress
Nurses treating COVID-19 in Wuhan were stressed. Lack of siblings, work 


\section{Discussion}

The present research aimed to analyze the psychological problems of nurses during the COVID19 pandemic. Among all academic papers selected and reviewed in this research, nine were crosssectional in type and explored psychological problems via questionnaires. The ever-expanding pandemic of the disease made the researchers use online surveys as emails or posts shared on social networks. One paper was qualitative in type and adopted a phenomenological approach. It used indepth semi-structured interviews to explain the psychological problems of nurses. It should be mentioned that seven papers were carried out in China. As the incidence of the disease began in China, more academic research has been conducted in this country followed by Italy, Pakistan, India, and Singapore.

The most common psychological problems that the nurses suffered from were panic, anxiety, stress, sleep disorder, discomfort, depression, lack of selfcontrol, overstimulation, and post-traumatic stress disorder. Emergence of negative feelings occurred faster than positive feelings among nurses (7). Additionally, women showed more symptoms, such as depression and anxiety, compared to men (3). Moreover, lower age and female gender had a significant effect on the incidence of depression, anxiety, and stress $(6,8,14)$. Concerns about financial problems and waiting for acknowledgments from superior ranks were also confirmed to affect the mental health of medical staff (13). In the body of research performed in China on this subject, being an only child was a background feature in question and was shown to be significantly involved in creating mental problems for the medical staff $(9,15)$.

Provision of personal protection facilities plays a key role in maintaining the mental health of nurses. Given the epidemic of the disease worldwide, attempts should be made to mentally support the healthcare staff and make the required interventions, such as holding adjusted debriefing sessions and psychological discharge practices, including hot-wash and psychological recovery. Based on the reviewed studies, age- and gender-specific interventions are required in this occupation as well (6). Furthermore, attention should be paid to sociocultural factors, such as the norm of having one child in China $(9,15)$ in the development of localized strategies and protocols to support the medical staff.

It has been about five months since the emergence of COVID-19 worldwide and copious research has been conducted on the clinical and psychological symptoms of the disease. Home quarantine and some other social obligations have induced certain psychological problems for people. These all have influenced nurses and other healthcare workers who have direct contact with patients and are also expected to take care of themselves not to transmit the disease to their family members. Based on the findings of the present systematic review of the related literature, systematic multipurpose interventions are required to support the healthcare staff psychologically. During the epidemic, specialized consultations can help reduce the fear of transmitting the infection among the healthcare staff, especially nurses. Such consultations also help them increase their self-confidence and stay positive.

As all studies on the psychological problems of nurses were cross-sectional in type, longitudinal studies are recommended to be conducted and certain interventions have to be executed to find optimal strategies to lower the psychological pressure on nurses during the pandemic. Longitudinal research can contribute to unpredictable future epidemics as well. Moreover, several suggestions have been made to promote the mental health of nurses: 1 . education of the nurses, 2. promotion of the psychical and mental tolerance of the nurses, 3. provision of full personal protection equipment, 4. development of social and psychological protocols to support nurses, 5 . reduction of work shifts, and 6. presence of psychologists and clergymen in the hospital. It should be mentioned that one limitation of the present research was the exclusion of articles that had been written in languages other than English.

\section{Conclusion}

During the epidemic and pandemic of infectious diseases, such as COVID-19, the healthcare staff, especially nurses experience various psychological problems. As nurses are on the frontier of fighting COVID-19, they require extensive support. In this context, health policymakers can plan appropriate educational programs and develop psychological support protocols to maintain and promote nurses' mental health.

\section{Acknowledgments}

The authors would like to thank Ms. A. Keivanshekouh at the Research Improvement Center of Shiraz University of Medical Sciences, Shiraz, Iran for improving the English of the manuscript.

\section{Footnotes}

Authors' Contributions: Milad Ahmadi Marzaleh, Ahmad Soltani, and Batoul Khoundabi were responsible for the study conception and design. Milad Ahmadi Marzaleh, Ahmad Soltani, and Batoul Khoundabi searched the relevant databases and included the appropriate articles according to the study objective. At the same time, Milad Ahmadi Marzaleh, Ahmad Soltani, and Batoul Khoundabi supervised the whole thesis. Milad Ahmadi Marzaleh, 
Ahmad Soltani, and Batoul Khoundabi prepared the first draft of the manuscript. Milad Ahmadi Marzaleh, Ahmad Soltani, and Batoul Khoundabi did the data analysis, made critical revisions to the paper for important intellectual content, and supervised the study. All authors read and approved the final manuscript

Conflict of Interest: The authors have no conflict of interest to declare.

Informed Consent: Nil.

Funding/Support: None declared.

Financial Disclosure: None declared.

\section{References}

1. Huang $\mathrm{C}$, Wang $\mathrm{Y}$, Li $\mathrm{X}$, Ren $\mathrm{L}$, Zhao J, Hu Y, et al. Clinical features of patients infected with 2019 novel coronavirus in Wuhan, China. Lancet. 2020;395(10223):497-506. doi: 10.1016/S0140-6736(20)30183-5. [PubMed: 31986264].

2. Maben J, Bridges J. Covid-19: Supporting nurses' psychological and mental health. J Clin Nurs. 2020;29(15-16):2742-50. doi: 10.1111/jocn.15307. [PubMed: 32320509].

3. Lai J, Ma S, Wang Y, Cai Z, Hu J, Wei N, et al. Factors associated with mental health outcomes among health care workers exposed to coronavirus disease 2019. JAMA Network Open. 2020; 3(3):e203976. doi: 10.1001/jamanetworkopen.2020.3976. [PubMed: 32202646].

4. Lee AM, Wong JG, McAlonan GM, Cheung V, Cheung C, Sham PC, et al. Stress and psychological distress among SARS survivors 1 year after the outbreak. Can J Psychiatry. 2007;52(4):233-40. doi: 10.1177/070674370705200405. [PubMed: 17500304].

5. Zhang C, Yang L, Liu S, Ma S, Wang Y, Cai Z, et al. Survey of insomnia and related social psychological factors among medical staff involved in the 2019 novel coronavirus disease outbreak. Front Psychiatry. 2020;11:306. doi: 10.3389/ fpsyt.2020.00306. [PubMed: 32346373].

6. Chew NW, Lee GK, Tan BY, Jing M, Goh Y, Ngiam NJ, et al. A multinational, multicentre study on the psychological outcomes and associated physical symptoms amongst healthcare workers during COVID-19 outbreak. Brain Behav Immun. 2020;88:559-65. doi: 10.1016/j.bbi.2020.04.049.
[PubMed: 32330593].

7. Sun N, Wei L, Shi S, Jiao D, Song R, Ma L, et al. A qualitative study on the psychological experience of caregivers of COVID19 patients. Am J Infect Control. 2020;48(6):592-8. doi: 10.1016/j.ajic.2020.03.018. [PubMed: 32334904].

8. Rossi R, Socci V, Pacitti F, Mensi S, Di Marco A, Siracusano A, et al. Mental Health outcomes among healthcare workers and the general population during the COVID-19 in Italy. Front Psychol. 2020;11:608986. doi: 10.3389/fpsyg.2020.608986. [PubMed: 33363500].

9. Mo Y, Deng L, Zhang L, Lang Q, Liao C, Wang N, et al. Work stress among Chinese nurses to support Wuhan in fighting against COVID-19 epidemic. J Nurs Manag. 2020;28(5):1002-9. doi: 10.1111/jonm.13014. [PubMed: 3225522].

10. Tang C, Liu C, Fang P, Xiang Y, Min R. Work-related accumulated fatigue among doctors in tertiary hospitals: a cross-sectional survey in six provinces of China. Int J Environ Res Public Health. 2019;16(17):3049. doi: 10.3390/ijer ph16173049. [PubMed: 31443480].

11. Amin S. The psychology of coronavirus fear: Are healthcare professionals suffering from corona-phobia? Int J Healthcare Manag. 2020;13(3):249-56. doi: 10.1080/20479700.2020. 1765119.

12. Zhu J, Sun L, Zhang L, Wang H, Fan A, Yang B, et al. Prevalence and influencing factors of anxiety and depression symptoms in the first-line medical staff fighting against COVID-19 in Gansu. Front Psychiatry. 2020;11:386. doi: 10.3389/fpsyt 2020.00386. [PubMed: 32411034].

13. Cai H, Tu B, Ma J, Chen L, Fu L, Jiang Y, et al. Psychological impact and coping strategies of frontline medical staff in Hunan between January and March 2020 during the outbreak of coronavirus disease 2019 (COVID-19) in Hubei, China. Med Sci Monit. 2020;26:e924171. doi: 10.12659/MSM.924171. [PubMed: 32291383].

14. Yin Q, Sun Z, Liu T, Ni X, Deng X, Jia Y, et al. Posttraumatic stress symptoms of health care workers during the corona virus disease 2019 (COVID-19). Clin Psychol Psychother. 2020;27(3):384-95. doi: 10.1002/cpp.2477. [PubMed: 32415733]

15. Li G, Miao J, Wang H, Xu S, Sun W, Fan Y, et al. Psychological impact on women health workers involved in COVID-19 outbreak in Wuhan: a cross-sectional study. J Neurol Neurosurg Psychiatry. 2020;91(8):895-7. doi: 10.1136/jnnp-2020323134. [PubMed: 32366684]. 